\title{
Communication \\ A Novel PIFA/KOH Promoted Approach to Synthesize C2-arylacylated Benzothiazoles as Potential Drug Scaffolds
}

\author{
Xiao-Tong Sun, Zhi-Gang Hu, Zhen Huang, Ling-Li Zhou and Jian-Quan Weng *
}

check for

updates

Citation: Sun, X.-T.; Hu, Z.-G.;

Huang, Z.; Zhou, L.-L.; Weng, J.-Q.

A Novel PIFA/KOH Promoted

Approach to Synthesize

C2-arylacylated Benzothiazoles as

Potential Drug Scaffolds. Molecules

2022, 27, 726. https://doi.org/

$10.3390 /$ molecules 27030726

Academic Editors: Graeme Barker and Simona Rapposelli

Received: 30 December 2021

Accepted: 21 January 2022

Published: 22 January 2022

Publisher's Note: MDPI stays neutral with regard to jurisdictional claims in published maps and institutional affiliations.

Copyright: (C) 2022 by the authors. Licensee MDPI, Basel, Switzerland. This article is an open access article distributed under the terms and conditions of the Creative Commons Attribution (CC BY) license (https:// creativecommons.org/licenses/by/ $4.0 /)$.
College of Chemical Engineering, Zhejiang University of Technology, Hangzhou 310014, China; SXT11096@163.com (X.-T.S.); H1718619378@163.com (Z.-G.H.); zjuthuangzhen@163.com (Z.H.); zl17398375385@163.com (L.-L.Z.)

* Correspondence: jqweng@zjut.edu.cn

\begin{abstract}
To discover an efficient and convenient method to synthesize C2-arylacylated benzothiazoles as potential drug scaffolds, a novel [bis(trifluoroacetoxy)iodo]benzene(PIFA)/ $\mathrm{KOH}$ synergistically promoted direct ring-opening $\mathrm{C} 2$-arylacylation reaction of $2 \mathrm{H}$-benzothiazoles with aryl methyl ketones has been developed. Various substrates were tolerated under optimized conditions affording the C2-arylacylation products in 70-95\% yields for 38 examples. A plausible mechanism was also proposed based on a series of controlled experiments.
\end{abstract}

Keywords: $2 \mathrm{H}$-benzothiazoles; aryl methyl ketones; arylacylation; PIFA/KOH

\section{Introduction}

Benzothiazole skeletons exist as key building blocks in natural products, drugs and agrochemicals and exhibit potent and diverse biological activities [1-4]. The benzothiazole scaffold is considered to be one of the most important substructures for drug discovery due to its various biological effects, including anti-cancer, anti-oxidant, anti-inflammatory, anti-microbial, anti-fungal, anti-convulsant, and anti-viral activities [5-11]. Among them, C2-arylacylated benzothiazole derivatives have attracted considerable attention in recent years due to their great potential as new drug candidates. For instance, as shown in Figure 1, 6-hydroxybenzothiophene ketone A exhibits potential for the treatment of breast cancer, endometriotic tissues, and other diseases by functioning as an inhibitor of 17b-hydroxysteroid dehydrogenase Type 1 (17b-HSD1) [12-14]. C2-arylacylated benzothiazole $\mathbf{B}$ act as a novel structural class of $\mathrm{Ca}^{2+} /$ calmodulin-dependent protein kinase II (CaMKII) inhibitors with the potential to be developed as anti-inflammatory agents [15]; 2-Benzothiazolyl-phenylmethanone $\mathbf{C}$ were found to be potent fatty acid amide hydrolase (FAAH) inhibitors with beneficial effects for disorders such as pain and inflammation [16]. As a potent inhibitor of antiapoptotic Bcl-2 proteins, acylpyrogallol D inhibited growth and induced apoptosis in human breast and prostate cancer cell lines [17]. Keto-benzothiazole E acted as a potent antiproliferative agent for melanoma [18], while $\mathbf{F}$ can potentially treat schizophrenia by inhibiting PDE10A [19].

Given their extensive potential for therapeutic use, the development of efficient synthetic strategies for C2-arylacylated benzothiazoles is an attractive research topic. Up to now, several methods have been investigated for the synthesis of C2-arylacylated benzothiazoles, including cyclization with or without the sulfuration of ortho-substituted anilines [20-23] and $\mathrm{sp}^{2} \mathrm{C}-\mathrm{H}$ bond functionalization of $2 \mathrm{H}$-benzothiazoles. From the perspective of synthetic simplicity and atom economy, the direct C2-functionalization method is relatively advantageous. Great efforts have been devoted to preparing C2-arylacylated benzothiazoles by the direct $\mathrm{C} 2$-functionalization of $2 \mathrm{H}$-benzothiazoles with aryl methyl ketones [24-27], phenylglyoxal [28], $\alpha$-oxocarboxylic acids [29,30], benzoic acid [31], and benzoyl chloride [32]. In particular, the direct C2-arylacylation of $2 \mathrm{H}$-benzothiazoles with aryl methyl ketones has generated great interest due to many "readily available" 
and "inexpensive" aryl methyl ketone analogs. In 2013, Deng and co-workers [24] reported the C2-arylacylation of $2 \mathrm{H}$-benzothiazoles using $\mathrm{O}_{2}$ as the oxidant in the presence of $\mathrm{FeCl}_{3} \cdot 6 \mathrm{H}_{2} \mathrm{O} / \mathrm{P}(\mathrm{Cy})_{3} \mathrm{HBF}_{4}$ at $120{ }^{\circ} \mathrm{C}$ (Figure 2a). In 2014, a $\mathrm{FeCl}_{3} \cdot 6 \mathrm{H}_{2} \mathrm{O}$-catalyzed C2arylacylated reaction of $2 \mathrm{H}$-benzothiazoles using $\mathrm{K}_{2} \mathrm{~S}_{2} \mathrm{O}_{8}$ as an oxidant was reported by $\mathrm{Yu}$ and co-workers [25] (Figure 2b). The same year, Song and co-workers [26] reported the CuIcatalyzed C2-arylacylation of $2 \mathrm{H}$-benzothiazoles under a nitrogen atmosphere (Figure 2c). In 2020, Ablajan and co-workers [27] developed a protocol for the C2-arylacylation of $2 \mathrm{H}$-benzothiazoles in the presence of $\mathrm{I}_{2}$ and TBHP (Figure 2d). Despite these notable advances, they still suffered from certain limitations such as relatively high reaction temperature, the transitional metal catalysts and inevitable metal residues, the use of the strongly corrosive additive $\mathrm{HBF}_{4}$, or the expensive ligand $\mathrm{P}(\mathrm{Cy})_{3} \mathrm{HBF}_{4}$. In addition, flammable and explosive organic peroxides were used as oxidizing reagents.

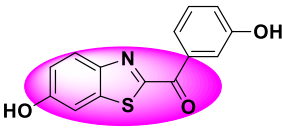

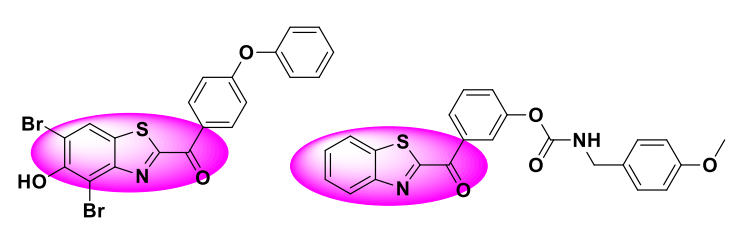

B

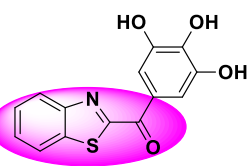

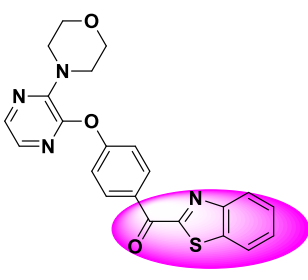

Figure 1. Several C2-arylacylated benzothiazole derivatives with potential as drug candidates.

This work

Previous work

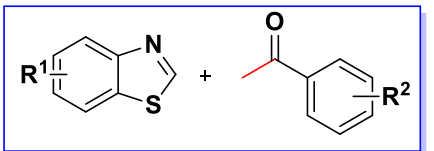

a) Deng and co-workers

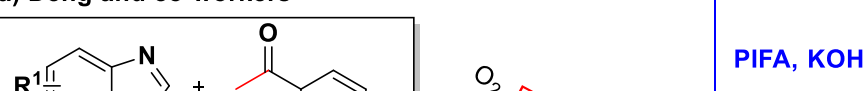

$\left.R^{1} \frac{\sqrt{1}}{4}\right\rangle+\frac{1}{s} R^{2}$

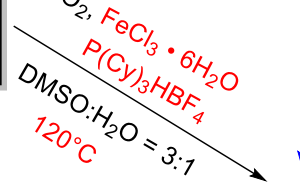

c) Song and co-workers

c)<smiles>c1ccc2scnc2c1</smiles>

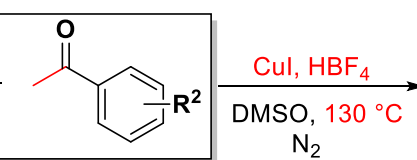

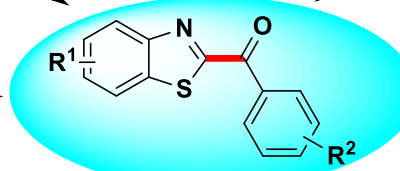

DMSO: $\mathrm{H}_{2} \mathrm{O}=3: 1$

$85^{\circ} \mathrm{C}$ b) Yu and co-workers

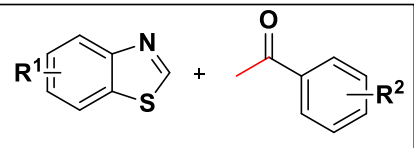

d) Ablajan and co-workers

Figure 2. Representative arylacylation reactions of $2 \mathrm{H}$-benzothiazoles.

In recent years, PIFA has received significant attention as a mild, low-toxic, and selective reagent in organic synthesis, which can also be used as an effective alternative to toxic, heavy metal-based oxidants, and expensive organometallic catalysts [33-35]. In line with our continuous efforts to extend the C2-functionalization methods for $2 \mathrm{H}$-benzothiazoles [36-40], herein we report a novel, convenient, and efficient PIFA/ $\mathrm{KOH}$ method which synergistically promotes the $\mathrm{C} 2$-arylacylation of $2 \mathrm{H}$-benzothiazoles with aryl methyl ketones, affording a wide variety of 2-arylacylated products with good yields. Our process also has the advantages of mild reaction conditions and being transitional metal-free. 


\section{Results and Discussion}

\subsection{Optimization of Reaction Conditions}

Initially, we chose $2 \mathrm{H}$-benzothiazole (1a) and acetophenone (2a) as the model substrates to investigate the reaction conditions. The results are outlined in Table 1 . Our examination of a series of common solvents demonstrated that the reactions led to the desired arylacylated product with a $7 \%$ yield in DMSO, while no product was produced in $\mathrm{MeCN}, \mathrm{DMF}$, and $\mathrm{H}_{2} \mathrm{O}$ (Table 1, entries 1-4). To our delight, the reaction efficiency was greatly improved when DMSO was mixed with $\mathrm{H}_{2} \mathrm{O}$. The experimental results indicated that the product yield reached $60 \%$ in the mixture of DMSO and $\mathrm{H}_{2} \mathrm{O}$ with a volume ratio of 3:1 (Table 1, entries 5-8). In addition, the screening of different bases showed that $\mathrm{KOH}$ provided the greatest yield (75\%) of the desired product (Table 1, entries 7 and 9-11). From the results in entries 11-14, it can be deduced that the base is necessary, with the optimal amount of $\mathrm{KOH}$ being one equivalent of $\mathbf{2 a}$. Furthermore, we changed the amount of PIFA, but poorer yields were obtained in all cases (Table 1, entries 16-18), and no product was observed in the absence of PIFA (Table 1, entry 15). Further investigation indicated that the temperature is very important for this transformation; the yield declined when the reaction temperature was decreased to $75^{\circ} \mathrm{C}$ or increased to $95^{\circ} \mathrm{C}$ (Table 1 , entries 19-20). When the reaction time increased from $8 \mathrm{~h}$ to $10 \mathrm{~h}$, the yield of 3aa was increased to $86 \%$, but no significant improvement was observed when the reaction time was increased to $12 \mathrm{~h}$ (Table 1, entries 21-22). Thus, the optimal reaction conditions involved PIFA (2 eq.) and $\mathrm{KOH}$ (1 eq.) in $\mathrm{DMSO} / \mathrm{H}_{2} \mathrm{O}(3: 1, v / v)$ at $85^{\circ} \mathrm{C}$ for $10 \mathrm{~h}$ (Table 1 , entry 21$)$.

Table 1. Optimization of reaction conditions ${ }^{a}$.

\begin{tabular}{|c|c|c|c|c|}
\hline Entry & Oxidant (eq.) & Base (eq.) & Solvent (mL) & Yield $(\%)^{b}$ \\
\hline 1 & PIFA (2) & $\mathrm{NaOH}(1)$ & DMSO (2) & 7 \\
\hline 2 & PIFA (2) & $\mathrm{NaOH}(1)$ & $\mathrm{MeCN}(2)$ & N.D. \\
\hline 3 & PIFA (2) & $\mathrm{NaOH}(1)$ & $\operatorname{DMF}(2)$ & N.D. \\
\hline 4 & PIFA (2) & $\mathrm{NaOH}(1)$ & $\mathrm{H}_{2} \mathrm{O}(2)$ & N.D. \\
\hline 5 & PIFA (2) & $\mathrm{NaOH}(1)$ & $\mathrm{DMSO} / \mathrm{H}_{2} \mathrm{O} 1: 1=(2)$ & 36 \\
\hline 6 & PIFA (2) & $\mathrm{NaOH}(1)$ & $\mathrm{DMSO} / \mathrm{H}_{2} \mathrm{O} 2: 1=(2)$ & 48 \\
\hline 7 & PIFA (2) & $\mathrm{NaOH}(1)$ & $\mathrm{DMSO} / \mathrm{H}_{2} \mathrm{O} 3: 1=(2)$ & 60 \\
\hline 8 & PIFA (2) & $\mathrm{NaOH}(1)$ & $\mathrm{DMSO} / \mathrm{H}_{2} \mathrm{O} 4: 1=(2)$ & 53 \\
\hline 9 & PIFA (2) & $\mathrm{K}_{2} \mathrm{CO}_{3}(1)$ & $\mathrm{DMSO} / \mathrm{H}_{2} \mathrm{O} 3: 1=(2)$ & 35 \\
\hline 10 & PIFA (2) & $\mathrm{Na}_{2} \mathrm{CO}_{3}(1)$ & $\mathrm{DMSO} / \mathrm{H}_{2} \mathrm{O} 3: 1=(2)$ & 32 \\
\hline 11 & PIFA (2) & $\mathrm{KOH}(1)$ & $\mathrm{DMSO} / \mathrm{H}_{2} \mathrm{O} 3: 1=(2)$ & 75 \\
\hline 12 & PIFA (2) & none & $\mathrm{DMSO} / \mathrm{H}_{2} \mathrm{O} 3: 1=(2)$ & N.D. \\
\hline 13 & PIFA (2) & $\mathrm{KOH}(0.5)$ & $\mathrm{DMSO} / \mathrm{H}_{2} \mathrm{O} 3: 1=(2)$ & 48 \\
\hline 14 & PIFA (2) & $\mathrm{KOH}(1.5)$ & $\mathrm{DMSO} / \mathrm{H}_{2} \mathrm{O} 3: 1=(2)$ & 53 \\
\hline 15 & none & $\mathrm{KOH}(1)$ & $\mathrm{DMSO} / \mathrm{H}_{2} \mathrm{O} 3: 1=(2)$ & N.D. \\
\hline 16 & PIFA (0.5) & $\mathrm{KOH}(1)$ & $\mathrm{DMSO} / \mathrm{H}_{2} \mathrm{O} 3: 1=(2)$ & 26 \\
\hline 17 & PIFA (1.5) & $\mathrm{KOH}(1)$ & $\mathrm{DMSO} / \mathrm{H}_{2} \mathrm{O} 3: 1=(2)$ & 38 \\
\hline 18 & PIFA (2.5) & $\mathrm{KOH}(1)$ & $\mathrm{DMSO} / \mathrm{H}_{2} \mathrm{O} 3: 1=(2)$ & 51 \\
\hline $19^{c}$ & PIFA (2) & $\mathrm{KOH}(1)$ & $\mathrm{DMSO} / \mathrm{H}_{2} \mathrm{O} 3: 1=(2)$ & 58 \\
\hline $20^{d}$ & PIFA (2) & $\mathrm{KOH}(1)$ & $\mathrm{DMSO} / \mathrm{H}_{2} \mathrm{O} 3: 1=(2)$ & 65 \\
\hline $21^{\mathrm{e}}$ & PIFA (2) & $\mathrm{KOH}(1)$ & $\mathrm{DMSO} / \mathrm{H}_{2} \mathrm{O} 3: 1=(2)$ & 86 \\
\hline $22^{f}$ & PIFA (2) & $\mathrm{KOH}(1)$ & $\mathrm{DMSO} / \mathrm{H}_{2} \mathrm{O} 3: 1=(2)$ & 85 \\
\hline
\end{tabular}

a Reaction conditions: 1a $(1.5$ eq., $0.45 \mathrm{mmol})$, 2a $(0.30 \mathrm{mmol})$, oxidant, base in solvent at $85^{\circ} \mathrm{C}$ for $8 \mathrm{~h}^{\mathrm{b}}{ }^{\mathrm{i}}$ isolated yield. ${ }^{\mathrm{c}} 75^{\circ} \mathrm{C} .{ }^{\mathrm{d}} 95^{\circ} \mathrm{C} .{ }^{\mathrm{e}} 10 \mathrm{~h} .{ }^{\mathrm{f}} 12 \mathrm{~h}$. 
Notably, the procedure was applicable to a $10 \mathrm{mmol}$ scale $(1.20 \mathrm{~g})$, and the product 3aa was isolated in 79\% (1.89 g) yield under the optimized reaction conditions (Figure 3).

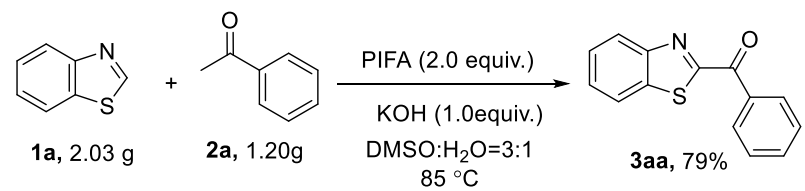

Figure 3. Gram-scale synthesis.

\subsection{Expansion of Substrate Scope}

With these optimized reaction conditions in mind, the scope of aryl methyl ketones (2) was first explored for the arylacylation of $2 \mathrm{H}$-benzothiazole (1a). As shown in Figure 4, the reactions with aryl methyl ketones bearing a methyl group at ortho-, meta-, and parapositions gave the corresponding arylacylated products in $80-85 \%$ yields (Figure $4 \mathrm{a}$, 3ab-3ad). The halogenated acetophenones (2-F, 3-F, 4-F, 2-Cl, 3-Cl, 4-Cl, 2-Br,3-Br, 4-Br, 2-I, $3-\mathrm{I}$, and $4-\mathrm{I}$ ) produced corresponding products in $70-88 \%$ yield (Figure $4 \mathrm{a}, 3 \mathbf{3 a e}-3 \mathrm{ap}$ ). In addition, 4-butylacetophenone, 4-methoxyacetophenone, 4-phenylacetophenone, and 2acetonaphthalene all formed corresponding arylacylated products in high yields of 86-95\% (Figure 4a, 3aq-3at). The above results demonstrated that a wide variety of aryl methyl ketones bearing either electron-donating or electron-withdrawing groups were well tolerated in this reaction.

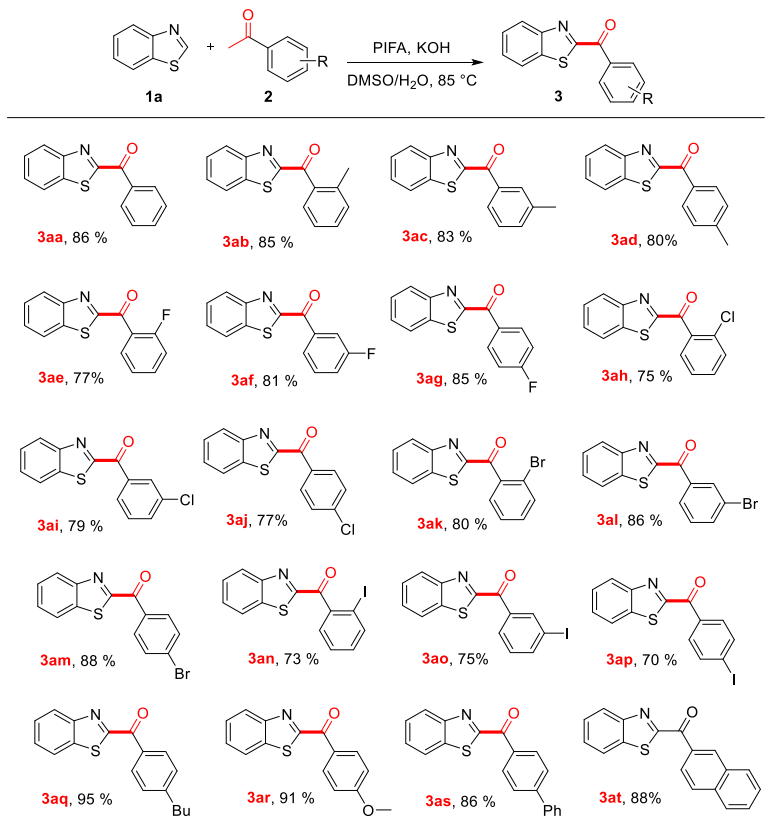

(a)

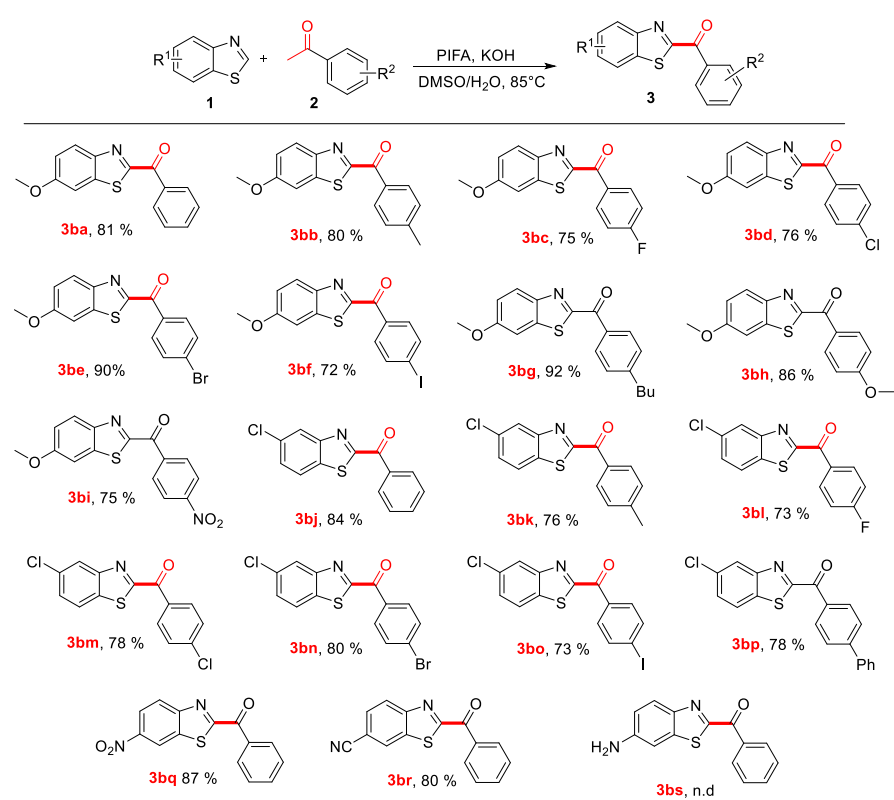

(b)

Figure 4. (a) Scope of substituted acetophenones. a a Reaction conditions: 1a (1.5 eq., $0.45 \mathrm{mmol})$, $2(0.30 \mathrm{mmol})$, PIFA (2.0 eq., $0.60 \mathrm{mmol}), \mathrm{KOH}$ (1.0 eq., $0.30 \mathrm{mmol}), \mathrm{DMSO} / \mathrm{H}_{2} \mathrm{O}(v / v, 3 / 1,2 \mathrm{~mL})$, $85^{\circ} \mathrm{C}, 10 \mathrm{~h}$; (b) Scope of substituted benzothiazoles and substituted acetophenones. ${ }^{\mathrm{b} b}$ Reaction conditions: 1 (1.5 eq., $0.45 \mathrm{mmol}), 2$ ( $0.30 \mathrm{mmol})$, PIFA (2.0 eq., $0.60 \mathrm{mmol}), \mathrm{KOH}$ (1.0eq., $0.30 \mathrm{mmol})$, $\mathrm{DMSO} / \mathrm{H}_{2} \mathrm{O}(v / v, 3 / 1,2 \mathrm{~mL}), 85^{\circ} \mathrm{C}, 10 \mathrm{~h}$.

Our scope was also extended to substituted $2 \mathrm{H}$-benzothiazoles and substituted aryl methyl ketones to investigate the utility and limits of the reaction (Figure $4 \mathrm{~b}$ ). To our delight, the reactions of 6-methoxybenzothiazole and 5-chlorobenzothiazole with aryl methyl ketones containing either electron-donating methyl and $n$-butyl groups or electronwithdrawing halogens; including fluoro, chloro, bromo, or iodo moieties or in addition to phenyl groups were well tolerated. These reactions all afforded corresponding arylacylated products in $72-92 \%$ yields (Figure 4 b, 3ba-bp). Meanwhile, 6-nitrobenzothiazole 
and 6-benzothiazolecarbonitrile were also tolerated by the reaction conditions, producing corresponding products $\mathbf{3 b q}$ and $3 \mathbf{b r}$ in $87 \%$ and $80 \%$ yield, respectively. The results demonstrated that the $2 \mathrm{H}$-benzothiazoles bearing electron-withdrawing or electron-donating groups were compatible with a variety of aryl methyl ketones. Unfortunately, the desired reaction did not occur between 6-aminobenzothiazole and acetophenone (3bs), presumably because the amino group is readily oxidizable.

\subsection{Mechanism Study}

In addition, a series of controlled experiments were used to explore the reaction mechanism as follows: (A) Two equivalents of the radical trap 2,2,6,6-tetramethyl-1-piperidinyloxy (TEMPO) were added to the mixture under optimal reaction conditions (Figure 5a). TEMPO, a known radical scavenger, will intercept the free radicals generated by the reaction, thus inhibiting the progress of the reaction [27]. The results indicated that the reaction was almost completely inhibited by TEMPO, with only a trace amount of 3 aa being detected. (B) The reaction mechanism behind the ring-opening of the $2 H$-benzothiazole was explored. The $2 \mathrm{H}$-benzothiazole was transformed into 2 -aminothiophenol (1b) in $83 \%$ yield under standard conditions (Figure 5b). (C) The reaction between 2-aminothiophenol (1b) and acetophenone (2a), under optimized reaction conditions, formed the target product (3aa) in $87 \%$ yield, which was close to that of the reaction between $2 \mathrm{H}$-benzothiazole and acetophenone (86\%, Figure 4, 3aa). This confirmed that the transformation might proceed via a ring-opening pathway. (D) The reaction between $2 H$-benzothiazole (1a) and 2,2-dihydroxy-1-phenylethan-1-one (2b) afforded 3aa in 92\% yield (Figure 5d). This result demonstrated that acetophenone (2a) was oxidized to phenylglyoxal (2b). (E) The reaction of 2-aminothiophenol (1b) with 2,2-dihydroxy-1-phenylethan-1-one (2b) afforded the desired product 3aa in 94\% yield, suggesting that 2-aminothiophenol (1b) was the intermediate product (Figure 5e).

(a)

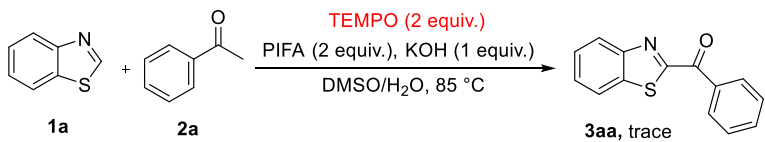

(b)
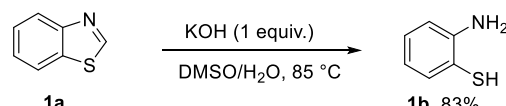

$1 \mathrm{~b}, 83 \%$ (d)
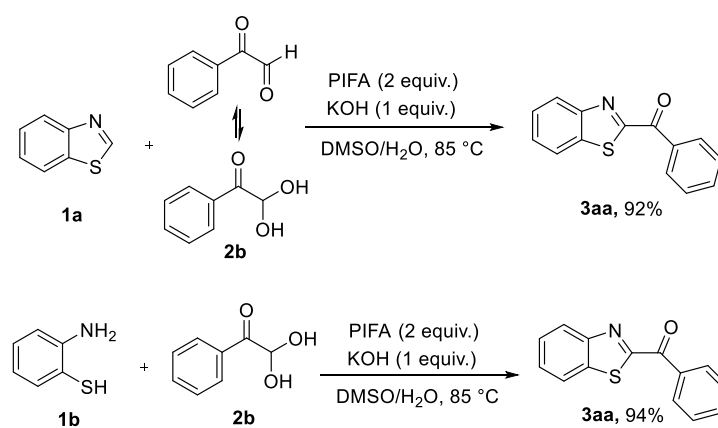

3aa, $87 \%$

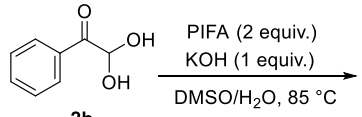

$2 \mathrm{~b}$

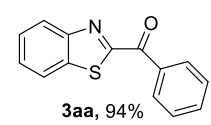

Figure 5. Mechanistic experiments.

Based on the above experimental results and related reports [24-27], a plausible mechanism was proposed in Figure 6. Initially, acetophenone (2a) is converted to A in the presence of PIFA, which is similar to the $\mathrm{I}_{2}$ promoted $\mathrm{sp}^{3} \mathrm{C}-\mathrm{H}$ functionalization [41]. Subsequently, A was further oxidized to phenylglyoxal (B) in the DMSO [28]. In the meantime, the ringopening of $2 \mathrm{H}$-benzothiazole (1a) under $\mathrm{KOH}$ produced 2-aminothiophenol (1) [ [42] The condensation of $\mathbf{1} \mathbf{b}$ with $\mathbf{B}$ formed an imine intermediate $\mathbf{C}$ [20-23], which could generate $\mathbf{D}$ through intramolecular cyclization [43]. Finally, the oxidative dehydrogenation of $\mathbf{D}$ furnished the target product 3aa through the elimination of $\mathrm{CF}_{3} \mathrm{COOH}$ and PhI. The presence of the latter compound was detected in the reaction solution by GC-MS [44,45]. 


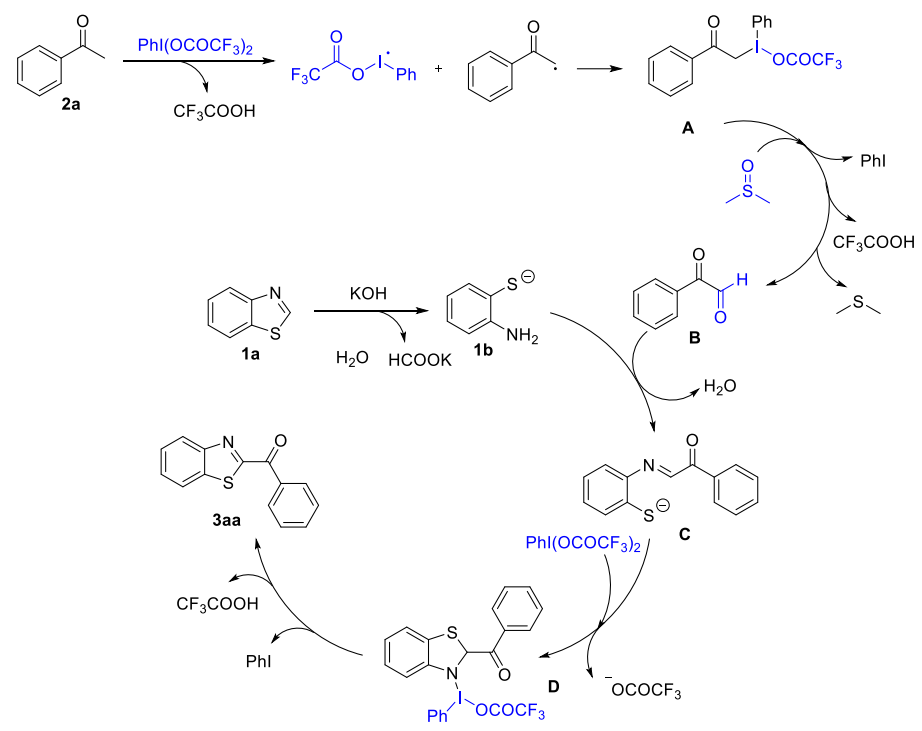

Figure 6. Plausible mechanism.

\section{Conclusions}

In summary, we have developed a novel PIFA/KOH synergistically promoted C2arylacylation of $2 \mathrm{H}$-benzothiazoles using aryl methyl ketones as carbonyl sources. This arylacylation reaction tolerates a wide range of functional groups affording 38 examples of the C2-arylacylated products in 70-95\% yield. This protocol provided an efficient and convenient method to synthesize C2-arylacylated benzothiazoles as potential drug scaffolds and complemented the existing approaches for the C2-arylacylation of aromatic rings.

\section{Materials and Methods}

\subsection{General Information}

All reactions were carried out under ambient atmosphere conditions in dried glassware. The reaction progress was monitored by TLC using silica gel GF254, and spots were visualized by exposure to UV light $(254 \mathrm{~nm})$. Melting points were determined using an X-4 apparatus without correction. NMR spectra were performed on a Bruker ADVANCE III instrument (500 MHz for ${ }^{1} \mathrm{H}-\mathrm{NMR}$ and $125 \mathrm{MHz}$ for ${ }^{13} \mathrm{C}-\mathrm{NMR}$ ) using TMS as an internal standard and $\mathrm{CDCl}_{3}$ or DMSO- $d_{6}$ as the solvent. The high-resolution mass spectra (HRMS) were determined using a Shimadzu LCMS-IT-TOF mass spectrometer equipped with an electrospray ionization (ESI) source.

\subsection{Synthesis}

A mixture of $2 \mathrm{H}$-benzothiazole $1(0.45 \mathrm{mmol})$, aryl methyl ketone $2(0.30 \mathrm{mmol}), \mathrm{KOH}$ (1 equiv., $0.30 \mathrm{mmol}$ ), and PIFA (2 equiv., $0.60 \mathrm{mmol}$ ) was heated at $85^{\circ} \mathrm{C}$ in $\mathrm{DMSO} / \mathrm{H}_{2} \mathrm{O}$ $(v / v, 3 / 1,2 \mathrm{~mL})$ for $10 \mathrm{~h}$. After cooling to room temperature, the reaction mixture was diluted with $\mathrm{H}_{2} \mathrm{O}(30 \mathrm{~mL})$ and extracted with dichloromethane $(3 \times 10 \mathrm{~mL})$. The combined organic layers were then dried over anhydrous $\mathrm{Na}_{2} \mathrm{SO}_{4}$, filtered, and concentrated under vacuum. The resulting residue was purified by column chromatography (silica gel, petroleum ether/ethyl acetate, 20:1 to 8:1) to give products $3 \mathbf{a a}-3 \mathbf{b s}$.

Benzo[d]thiazol-2-yl(phenyl)methanone (3aa): Yellow solid, yield 86\% (61 mg); m.p.: 98-99 ${ }^{\circ} \mathrm{C}$; ${ }^{1} \mathrm{H}$ NMR $\left(500 \mathrm{MHz}, \mathrm{CDCl}_{3}\right) \delta 8.58(\mathrm{dd}, J=8.0,1.0 \mathrm{~Hz}, 2 \mathrm{H}), 8.27(\mathrm{~d}, J=7.5 \mathrm{~Hz}, 1 \mathrm{H}), 8.04$ $(\mathrm{d}, J=7.0 \mathrm{~Hz}, 1 \mathrm{H}), 7.73-7.65(\mathrm{~m}, 1 \mathrm{H}), 7.64-7.54(\mathrm{~m}, 4 \mathrm{H}) ;{ }^{13} \mathrm{C} \mathrm{NMR}\left(125 \mathrm{MHz}, \mathrm{CDCl}_{3}\right) \delta$ 185.40, 167.17, 153.94, 137.06, 135.03, 133.91, 131.31, 128.53, 127.64, 126.95, 125.77, 122.19. These spectroscopic data correspond to the reported data in reference [27].

Benzo[d]thiazol-2-yl(o-tolyl)methanone (3ab): Yellow solid; yield 85\% (64 mg); m.p.: 110-112 ${ }^{\circ} \mathrm{C}$; ${ }^{1} \mathrm{H}$ NMR $\left(500 \mathrm{MHz}, \mathrm{CDCl}_{3}\right) \delta 8.21(\mathrm{dd}, J=9.0,1.5 \mathrm{~Hz}, 1 \mathrm{H}), 8.03(\mathrm{td}, J=7.5,1.0 \mathrm{~Hz}, 2 \mathrm{H})$, 7.60-7.54 (m, 2H), 7.52-7.48 (m, 1H), $7.37(\mathrm{t}, J=7.5 \mathrm{~Hz}, 2 \mathrm{H}), 2.55(\mathrm{~s}, 3 \mathrm{H}) ;{ }^{13} \mathrm{C} \mathrm{NMR}(125 \mathrm{MHz}$, 
$\left.\mathrm{CDCl}_{3}\right) \delta 189.27,167.61,153.86,139.11,137.2,135.25,132.03,131.58,131.37,127.69,126.95$, $125.86,125.35,122.26,20.68$. These spectroscopic data correspond to the reported data in reference [27].

Benzo[d]thiazol-2-yl(m-tolyl)methanone (3ac): Yellow solid; yield 83\% (63 mg); m.p.: $72-74{ }^{\circ} \mathrm{C}$; ${ }^{1} \mathrm{H} \mathrm{NMR}\left(500 \mathrm{MHz}, \mathrm{CDCl}_{3}\right) \delta 8.40(\mathrm{~d}, J=7.5 \mathrm{~Hz}, 1 \mathrm{H}), 8.30(\mathrm{~s}, 1 \mathrm{H}), 8.29-8.26(\mathrm{~m}, 1 \mathrm{H})$, 8.05-8.03 (m, 1H), 7.63-7.57 (m, 2H), 7.51-7.45 (m, 2H), 2.50 (s, 3H); ${ }^{13} \mathrm{C}$ NMR $(125 \mathrm{MHz}$, $\left.\mathrm{CDCl}_{3}\right) 185.69,167.29,153.94,138.35,137.06,135.07,134.76,131.50,128.69,128.43,127.59$, $126.91,125.78,122.19,21.47$. These spectroscopic data correspond to the reported data in reference [27].

Benzo[d]thiazol-2-yl(p-tolyl)methanone (3ad): Yellow solid; yield 80\% (61 mg); m.p.: 96-98 ${ }^{\circ} \mathrm{C}$; ${ }^{1} \mathrm{H}$ NMR $\left(500 \mathrm{MHz}, \mathrm{CDCl}_{3}\right) \delta 8.50(\mathrm{~d}, J=8.5 \mathrm{~Hz}, 1 \mathrm{H}), 8.31-8.14(\mathrm{~m}, 1 \mathrm{H}), 8.11-7.92(\mathrm{~m}, 1 \mathrm{H})$, $7.58(\mathrm{dtd}, J=21.0,7.0,1.5 \mathrm{~Hz}, 2 \mathrm{H}), 7.38(\mathrm{~d}, J=8.5 \mathrm{~Hz}, 1 \mathrm{H}), 2.49(\mathrm{~s}, 2 \mathrm{H}) ;{ }^{13} \mathrm{C}$ NMR $(125 \mathrm{MHz}$, $\left.\mathrm{CDCl}_{3}\right) \delta 184.94,153.94,153.94,137.01,132.47,131.44,129.28,127.49,126.85,125.69,122.16$, 21.85. These spectroscopic data correspond to the reported data in reference [27].

Benzo[d]thiazol-2-yl(2-fluorophenyl)methanone (3ae): Yellow solid; yield 77\% (59 mg); m.p.: 81-83 ${ }^{\circ} \mathrm{C} ;{ }^{1} \mathrm{H}$ NMR $\left(500 \mathrm{MHz}, \mathrm{CDCl}_{3}\right) \delta 8.20(\mathrm{~d}, J=7.3 \mathrm{~Hz}, 1 \mathrm{H}), 8.06-8.01(\mathrm{~m}, 2 \mathrm{H}), 7.64-7.55$ $(\mathrm{m}, 3 \mathrm{H}), 7.34(\mathrm{t}, J=7.9 \mathrm{~Hz}, 1 \mathrm{H}), 7.25(\mathrm{dd}, J=17.7,8.0 \mathrm{~Hz}, 1 \mathrm{H}) ;{ }^{13} \mathrm{C}$ NMR $\left(125 \mathrm{MHz}, \mathrm{CDCl}_{3}\right)$ $\delta 185.33,166.21,161.24(\mathrm{~d}, J=255.0 \mathrm{~Hz}), 153.72,137.29,134.41(\mathrm{~d}, J=8.8 \mathrm{~Hz}), 131.91,127.86$, $127.04,125.86,124.89(\mathrm{~d}, J=11.3 \mathrm{~Hz}), 124.00(\mathrm{~d}, J=3.75 \mathrm{~Hz}), 122.29,116.69(\mathrm{~d}, J=21.0 \mathrm{~Hz})$. These spectroscopic data correspond to the reported data in reference [26].

Benzo[d]thiazol-2-yl(3-fluorophenyl)methanone (3af): Yellow solid; yield 81\% (62 mg); m.p.: 78-80 ${ }^{\circ} \mathrm{C} ;{ }^{1} \mathrm{H}$ NMR $\left(500 \mathrm{MHz}, \mathrm{CDCl}_{3}\right) \delta 8.41(\mathrm{~d}, J=8.0 \mathrm{~Hz}, 1 \mathrm{H}), 8.35(\mathrm{ddd}, J=9.5,2.5$, $1.5 \mathrm{~Hz}, 1 \mathrm{H}), 8.28(\mathrm{~d}, J=7.0 \mathrm{~Hz}, 1 \mathrm{H}), 8.05(\mathrm{~d}, J=7.5 \mathrm{~Hz}, 1 \mathrm{H}), 7.64-7.55(\mathrm{~m}, 3 \mathrm{H}), 7.39$ (tdd, $J=8.0,2.5,1.0 \mathrm{~Hz}, 1 \mathrm{H}) ;{ }^{13} \mathrm{C}$ NMR $\left(125 \mathrm{MHz}, \mathrm{CDCl}_{3}\right) \delta 183.97,166.62,153.90,137.13,130.16$ $(\mathrm{d}, J=7.5 \mathrm{~Hz}), 127.89,127.11,125.90,122.23,120.95(\mathrm{~d}, J=21.3 \mathrm{~Hz}), 118.17(\mathrm{~d}, J=23.8 \mathrm{~Hz})$. These spectroscopic data correspond to the reported data in reference [26].

Benzo[d]thiazol-2-yl(4-fluorophenyl)methanone (3ag): Yellow solid; yield 85\% (65.6 mg); m.p.: 100-102 ${ }^{\circ} \mathrm{C} ;{ }^{1} \mathrm{H}$ NMR (500 MHz, $\left.\mathrm{CDCl}_{3}\right) \delta 8.70(\mathrm{dd}, J=8.5,5.5 \mathrm{~Hz}, 2 \mathrm{H}), 8.29-8.24(\mathrm{~m}$, $1 \mathrm{H}), 8.06-8.02(\mathrm{~m}, 1 \mathrm{H}), 7.60(\mathrm{dtd}, J=15.5,7.5,1.5 \mathrm{~Hz}, 2 \mathrm{H}), 7.26(\mathrm{t}, J=9.0 \mathrm{~Hz}, 2 \mathrm{H}) ;{ }^{13} \mathrm{C}$ $\operatorname{NMR}\left(125 \mathrm{MHz} \mathrm{CDCl}_{3}\right) \delta 183.56,167.06,166.42(\mathrm{~d}, J=256.0 \mathrm{~Hz}), 153.87,137.03,134.19$ $(\mathrm{d}, J=8.8 \mathrm{~Hz}), 131.32(\mathrm{~d}, J=2.5 \mathrm{~Hz}), 127.70,126.99,125.71,122.18,115.73(\mathrm{~d}, J=22.5 \mathrm{~Hz})$. These spectroscopic data correspond to the reported data in reference [26].

Benzo[d]thiazol-2-yl(2-chlorophenyl)methanone (3ah): Yellow solid; yield 75\% (61 mg); m.p.: 92-93 ${ }^{\circ} \mathrm{C} ;{ }^{1} \mathrm{H}$ NMR $\left(500 \mathrm{MHz}, \mathrm{CDCl}_{3}\right) \delta 8.21-8.17(\mathrm{~m}, 1 \mathrm{H}), 8.06-8.01(\mathrm{~m}, 1 \mathrm{H}), 7.80-7.76(\mathrm{~m}$, $1 \mathrm{H}), 7.62-7.49(\mathrm{~m}, 6 \mathrm{H}), 7.45(\mathrm{ddd}, J=7.5,6.0,2.5 \mathrm{~Hz}, 1 \mathrm{H}) ;{ }^{13} \mathrm{C} \mathrm{NMR}\left(125 \mathrm{MHz}, \mathrm{CDCl}_{3}\right) \delta$ 187.61, 165.95, 153.76, 137.45, 136.07, 132.63, 132.36, 130.79, 130.55, 127.97, 127.07, 126.49, 125.97, 122.33. These spectroscopic data correspond to the reported data in reference [26].

Benzo[d]thiazol-2-yl(3-chlorophenyl)methanone (3ai): Yellow solid; yield 79\% (64 mg); m.p.: $128-129{ }^{\circ} \mathrm{C} ;{ }^{1} \mathrm{H} \mathrm{NMR}\left(500 \mathrm{MHz}, \mathrm{CDCl}_{3}\right) \delta 8.58(\mathrm{t}, J=2.0 \mathrm{~Hz}, 1 \mathrm{H}), 8.50(\mathrm{~d}, J=8.0 \mathrm{~Hz}, 1 \mathrm{H}), 8.28$ $(\mathrm{d}, J=7.5 \mathrm{~Hz}, 1 \mathrm{H}), 8.05(\mathrm{~d}, J=7.5 \mathrm{~Hz}, 1 \mathrm{H}), 7.67-7.51(\mathrm{~m}, 4 \mathrm{H}) ;{ }^{13} \mathrm{C} \mathrm{NMR}\left(125 \mathrm{MHz}, \mathrm{CDCl}_{3}\right) \delta$ $184.05,166.48,153.86,137.11,136.50,134.73,133.80,131.19,129.82,129.45,127.90,127.11$, $125.91,122.22$. These spectroscopic data correspond to the reported data in reference [27].

Benzo[d]thiazol-2-yl(4-chlorophenyl)methanone (3aj): Yellow solid; yield 77\% (60 mg); m.p.: 99-101 ${ }^{\circ} \mathrm{C} ;{ }^{1} \mathrm{H}$ NMR $\left(500 \mathrm{MHz}, \mathrm{CDCl}_{3}\right) \delta 8.58(\mathrm{~d}, J=8.5 \mathrm{~Hz}, 2 \mathrm{H}), 8.26(\mathrm{~d}, J=7.5 \mathrm{~Hz}, 1 \mathrm{H}), 8.05$ $(\mathrm{d}, J=7.5 \mathrm{~Hz}, 1 \mathrm{H}), 7.64-7.54(\mathrm{~m}, 4 \mathrm{H}) ;{ }^{13} \mathrm{C} \mathrm{NMR}\left(125 \mathrm{MHz} \mathrm{CDCl}_{3}\right) \delta 184.06,166.86,153.89$, $140.67,137.10,133.33,132.76,128.91,127.82,127.08,125.80,122.23$. These spectroscopic data correspond to the reported data in reference [26].

Benzo[d]thiazol-2-yl(2-bromophenyl)methanone (3ak): Yellow solid; yield 80\% (76 mg); m.p.: 103-105 ${ }^{\circ} \mathrm{C} ;{ }^{1} \mathrm{H}$ NMR $\left(500 \mathrm{MHz}, \mathrm{CDCl}_{3}\right) \delta 8.19(\mathrm{~d}, J=9.5 \mathrm{~Hz}, 1 \mathrm{H}), 8.04(\mathrm{~d}, J=9.5 \mathrm{~Hz}, 1 \mathrm{H})$, 7.77-7.70 (m, 3H), 7.60-7.55 (m, 2H), $7.50(\mathrm{td}, J=7.5,1.0 \mathrm{~Hz}, 1 \mathrm{H}), 7.44(\mathrm{td}, J=8.0,2.0 \mathrm{~Hz}$, 
$1 \mathrm{H}) ;{ }^{13} \mathrm{C}$ NMR $\left(125 \mathrm{MHz}, \mathrm{CDCl}_{3}\right) \delta 188.31,165.67,153.76,138.09,137.50,133.70,132.37$, $130.71,128.00,127.07,125.99,122.34,120.64$. These spectroscopic data correspond to the reported data in reference [26].

Benzo[d]thiazol-2-yl(3-bromophenyl)methanone (3al): Yellow solid; yield 86\% (82 mg); m.p.: 98-100 ${ }^{\circ} \mathrm{C} ;{ }^{1} \mathrm{H}$ NMR $\left(500 \mathrm{MHz}, \mathrm{CDCl}_{3}\right) \delta 8.72(\mathrm{t}, J=2.0 \mathrm{~Hz}, 1 \mathrm{H}), 8.55(\mathrm{~d}, J=8.0 \mathrm{~Hz}, 1 \mathrm{H})$, $8.29(\mathrm{~d}, J=7.5 \mathrm{~Hz}, 1 \mathrm{H}), 8.05(\mathrm{~d}, J=7.5 \mathrm{~Hz}, 1 \mathrm{H}), 7.81(\mathrm{~d}, J=8.0 \mathrm{~Hz}, 1 \mathrm{H}), 7.64-7.57(\mathrm{~m}, 2 \mathrm{H})$, $7.47(\mathrm{t}, J=8.0 \mathrm{~Hz}, 1 \mathrm{H}) ;{ }^{13} \mathrm{C}$ NMR $\left(125 \mathrm{MHz}, \mathrm{CDCl}_{3}\right) \delta 183.99,136.70,134.05,129.99,127.91$, $127.11,125.93,122.68,122.22$. These spectroscopic data correspond to the reported data in reference [27].

Benzo[d]thiazol-2-yl(4-bromophenyl)methanone (3am): Yellow solid; yield 88\% (84 mg); m.p.: 92-94 ${ }^{\circ} \mathrm{C} ;{ }^{1} \mathrm{H}$ NMR $\left(500 \mathrm{MHz}, \mathrm{CDCl}_{3}\right) \delta 8.49(\mathrm{~d}, J=8.5 \mathrm{~Hz}, 2 \mathrm{H}), 8.25(\mathrm{~d}, J=7.5 \mathrm{~Hz}, 1 \mathrm{H}), 8.04$ $(\mathrm{d}, J=7.5 \mathrm{~Hz}, 1 \mathrm{H}), 7.72(\mathrm{~d}, J=8.5 \mathrm{~Hz}, 2 \mathrm{H}), 7.63-7.56(\mathrm{~m}, 2 \mathrm{H}) ;{ }^{13} \mathrm{C} \mathrm{NMR}\left(125 \mathrm{MHz}, \mathrm{CDCl}_{3}\right) \delta$ $184.27,166.80,153.88,137.10,133.74,132.80,131.89,129.53,127.83,127.08,125.81,122.23$. These spectroscopic data correspond to the reported data in reference [27].

Benzo[d]thiazol-2-yl(2-iodophenyl)methanone (3an): Yellow solid; yield 73\% (79 mg); m.p.: 121-123 ${ }^{\circ} \mathrm{C} ;{ }^{1} \mathrm{H}$ NMR $\left(500 \mathrm{MHz}, \mathrm{CDCl}_{3}\right) \delta 8.18(\mathrm{~d}, J=8.0 \mathrm{~Hz}, 1 \mathrm{H}), 8.08-8.04(\mathrm{~m}, 1 \mathrm{H}), 7.97$ $(\mathrm{d}, J=7.5 \mathrm{~Hz}, 1 \mathrm{H}), 7.74(\mathrm{dd}, J=8.0,2.0 \mathrm{~Hz}, 1 \mathrm{H}), 7.60-7.55(\mathrm{~m}, 1 \mathrm{H}), 7.52-7.44(\mathrm{~m}, 2 \mathrm{H}), 7.19$ $(\mathrm{td}, J=8.0,1.5 \mathrm{~Hz}, 1 \mathrm{H}) ;{ }^{13} \mathrm{C}$ NMR $\left(125 \mathrm{MHz}, \mathrm{CDCl}_{3}\right) \delta 167.98,140.66,136.18,131.52,131.30$, $128.25,126.40,125.59,123.77,121.57,96.35$. ESI-HRMS calcd $\mathrm{C}_{14} \mathrm{H}_{8} \mathrm{INOS}[\mathrm{M}+\mathrm{H}]^{+} 365.9450$; found, 365.9469 .

Benzo[d]thiazol-2-yl(3-iodophenyl)methanone (3ao): Yellow solid; yield 75\% (82 mg); m.p.: 105-107 ${ }^{\circ} \mathrm{C} ;{ }^{1} \mathrm{H}$ NMR $\left(500 \mathrm{MHz}, \mathrm{CDCl}_{3}\right) \delta 8.87(\mathrm{t}, J=1.5 \mathrm{~Hz}, 1 \mathrm{H}), 8.59(\mathrm{dt}, J=7.5,1.5$ $\mathrm{Hz}, 1 \mathrm{H}), 8.29-8.26(\mathrm{~m}, 1 \mathrm{H}), 8.04-7.99(\mathrm{~m}, 2 \mathrm{H}), 7.63-7.57(\mathrm{~m}, 2 \mathrm{H}), 7.32(\mathrm{t}, J=8.0 \mathrm{~Hz}, 1 \mathrm{H})$; ${ }^{13} \mathrm{C}$ NMR $\left(125 \mathrm{MHz}, \mathrm{CDCl}_{3}\right) \delta 183.82,166.36,153.82,142.53,139.79,137.08,136.69,130.50$, $130.12,127.87,127.07,125.90,122.19,94.02$. ESI-HRMS calcd $\mathrm{C}_{14} \mathrm{H}_{8} \mathrm{INOS}[\mathrm{M}+\mathrm{H}]^{+} 365.9450$; found, 365.9468 .

Benzo[d]thiazol-2-yl(4-iodophenyl)methanone (3ap): Yellow solid; yield 70\% (76 mg); m.p.: 113-115 ${ }^{\circ} \mathrm{C} ;{ }^{1} \mathrm{H}$ NMR (500 MHz, $\left.\mathrm{CDCl}_{3}\right) \delta 8.33-8.29(\mathrm{~m}, 2 \mathrm{H}), 8.27-8.23(\mathrm{~m}, 1 \mathrm{H}), 8.06-8.01$ $(\mathrm{m}, 1 \mathrm{H}), 7.97-7.93(\mathrm{~m}, 2 \mathrm{H}), 7.64-7.56(\mathrm{~m}, 2 \mathrm{H}) ;{ }^{13} \mathrm{C} \mathrm{NMR}\left(125 \mathrm{MHz}, \mathrm{CDCl}_{3}\right) \delta 184.58,166.73$, $153.83,137.87,137.07,134.23,132.56,127.80,127.05,125.78,122.20,102.57$. These spectroscopic data correspond to the reported data in reference [46].

Benzo[d]thiazol-2-yl(4-butylphenyl)methanone (3aq): Yellow liquid; yield 95\% (84 mg); ${ }^{1} \mathrm{H}$ NMR $\left(500 \mathrm{MHz} \mathrm{CDCl}_{3}\right) \delta 8.52(\mathrm{~d}, J=8.0 \mathrm{~Hz}, 2 \mathrm{H}), 8.31-8.19(\mathrm{~m}, 1 \mathrm{H}), 8.07-7.91(\mathrm{~m}, 1 \mathrm{H}), 7.62-7.52$ $(\mathrm{m}, 2 \mathrm{H}), 7.38(\mathrm{~d}, J=8.4 \mathrm{~Hz}, 2 \mathrm{H}), 2.78-2.70(\mathrm{~m}, 2 \mathrm{H}), 1.71-1.63(\mathrm{~m}, 2 \mathrm{H}), 1.46-1.36(\mathrm{~m}, 2 \mathrm{H})$, $1.02-0.91(\mathrm{~m}, 3 \mathrm{H}) ;{ }^{13} \mathrm{C}$ NMR $\left(125 \mathrm{MHz}, \mathrm{CDCl}_{3}\right) \delta 184.88,167.51,153.91,149.88,136.98$, $132.60,131.45,128.64,127.51,126.82,125.66,122.13,35.87,33.15,22.35,13.91$. ESI-HRMS calcd $\mathrm{C}_{18} \mathrm{H}_{8} \mathrm{NOS}[\mathrm{M}+\mathrm{H}]^{+}$296.1109; found, 296.1109 .

Benzo[d] thiazol-2-yl(4-methoxyphenyl)methanone (3ar): Yellow solid; yield 91\% (73 mg); m.p.: 126-128 ${ }^{\circ} \mathrm{C} ;{ }^{1} \mathrm{H}$ NMR (500 MHz, $\left.\mathrm{CDCl}_{3}\right) \delta$ 8.69-8.63 (m, 2H), 8.28-8.22 (m, 1H), 7.95-7.82 $(\mathrm{m}, 1 \mathrm{H}), 7.65-7.51(\mathrm{~m}, 2 \mathrm{H}), 7.04-6.98(\mathrm{~m}, 2 \mathrm{H}), 3.90(\mathrm{~s}, 3 \mathrm{H}) ;{ }^{13} \mathrm{C} \mathrm{NMR}\left(125 \mathrm{MHz}, \mathrm{CDCl}_{3}\right) \delta$ $183.41,167.91,164.42,153.91,136.90,133.85,127.80,127.35,126.77,125.53,122.11,113.89$, 55.55. These spectroscopic data correspond to the reported data in reference [26].

[1,1'-Biphenyl]-4-yl(benzo[d]thiazol-2-yl)methanone (3as): White solid; yield 86\% (81 mg); m.p.: 100-102 ${ }^{\circ} \mathrm{C} ;{ }^{1} \mathrm{H}$ NMR $\left(500 \mathrm{MHz}, \mathrm{CDCl}_{3}\right) \delta 8.68(\mathrm{~d}, J=8.5 \mathrm{~Hz}, 2 \mathrm{H}), 8.29(\mathrm{~d}, J=7.5 \mathrm{~Hz}, 1 \mathrm{H})$, $8.05(\mathrm{~d}, J=7.0 \mathrm{~Hz}, 1 \mathrm{H}), 7.81(\mathrm{~d}, J=8.0 \mathrm{~Hz}, 2 \mathrm{H}), 7.70(\mathrm{~d}, J=7.0 \mathrm{~Hz}, 2 \mathrm{H}), 7.63-7.57(\mathrm{~m}, 2 \mathrm{H})$, 7.55-7.49 (m, 2H), 7.47-7.42 (m, 1H); $\left.{ }^{13} \mathrm{C} \mathrm{NMR} \mathrm{(125} \mathrm{MHz,} \mathrm{CDCl}_{3}\right) \delta 184.78,167.35,153.93$, $146.56,139.90,137.04,133.71,131.89,128.99,128.38,127.60,127.37,127.17,126.93,125.73$, 122.18. These spectroscopic data correspond to the reported data in reference [46].

Benzo[d]thiazol-2-yl(naphthalen-2-yl)methanone (3at): Yellow solid; yield 88\% (76 mg); m.p.: 145-147 ${ }^{\circ} \mathrm{C} ;{ }^{1} \mathrm{H}$ NMR (500 MHz, $\left.\mathrm{CDCl}_{3}\right) \delta 9.36(\mathrm{~s}, 1 \mathrm{H}), 8.46(\mathrm{~d}, J=9.0 \mathrm{~Hz}, 1 \mathrm{H}), 8.32$ 
$(\mathrm{d}, J=7.5 \mathrm{~Hz}, 1 \mathrm{H}), 8.10(\mathrm{~d}, J=8.5 \mathrm{~Hz}, 1 \mathrm{H}), 8.00(\mathrm{~d}, J=8.5 \mathrm{~Hz}, 1 \mathrm{H}), 7.70-7.58(\mathrm{~m}, 4 \mathrm{H}) ;{ }^{13} \mathrm{C}$ NMR $\left(125 \mathrm{MHz}, \mathrm{CDCl}_{3}\right) \delta 185.07,167.41,153.96,137.04,135.98,134.36,132.48,132.24,130.24$, $129.02,128.34,127.78,127.58,126.91,126.73,125.83,125.76,122.18$. These spectroscopic data correspond to the reported data in reference [26].

(6-Methoxybenzo[d]thiazol-2-yl)(phenyl)methanone (3ba): Yellow solid; yield $81 \%$ (65 mg); m.p.: $138-140{ }^{\circ} \mathrm{C} ;{ }^{1} \mathrm{H}$ NMR $\left(500 \mathrm{MHz}, \mathrm{CDCl}_{3}\right) \delta 8.56(\mathrm{dd}, J=8.0,1.0 \mathrm{~Hz}, 2 \mathrm{H}), 8.12(\mathrm{~d}, J=9.0 \mathrm{~Hz}$, $1 \mathrm{H}), 7.67(\mathrm{t}, J=7.5 \mathrm{~Hz}, 1 \mathrm{H}), 7.57(\mathrm{t}, J=8.0 \mathrm{~Hz}, 2 \mathrm{H}), 7.43(\mathrm{~d}, J=2.5 \mathrm{~Hz}, 1 \mathrm{H}), 7.20(\mathrm{dd}$, $J=9.0,2.5 \mathrm{~Hz}, 1 \mathrm{H}), 3.95(\mathrm{~s}, 3 \mathrm{H}) ;{ }^{13} \mathrm{C}$ NMR $\left(125 \mathrm{MHz}, \mathrm{CDCl}_{3}\right) \delta 185.21,164.70,159.84,148.61$, $139.16,135.26,133.68,131.20,128.46,126.51,117.63,103.49,55.89$. These spectroscopic data correspond to the reported data in reference [36].

(6-Methoxybenzo[d] thiazol-2-yl)(p-tolyl)methanone (3bb): Yellow solid; yield 80\% (68 mg); m.p.: $153-155^{\circ} \mathrm{C} ;{ }^{1} \mathrm{H}$ NMR $\left(500 \mathrm{MHz}, \mathrm{CDCl}_{3}\right) \delta 8.48(\mathrm{~d}, J=8.0 \mathrm{~Hz}, 2 \mathrm{H}), 8.11(\mathrm{~d}, J=9.0 \mathrm{~Hz}$, $1 \mathrm{H}), 7.42(\mathrm{~d}, J=2.5 \mathrm{~Hz}, 1 \mathrm{H}), 7.37(\mathrm{~d}, J=8.0 \mathrm{~Hz}, 2 \mathrm{H}), 7.19(\mathrm{dd}, J=9.5,3.0 \mathrm{~Hz}, 1 \mathrm{H}), 3.94$ $(\mathrm{s}, 3 \mathrm{H}), 2.48(\mathrm{~s}, 3 \mathrm{H}) ;{ }^{13} \mathrm{C}$ NMR $\left(125 \mathrm{MHz}, \mathrm{CDCl}_{3}\right) \delta 184.72,164.98,159.67,148.54,144.71$, $139.03,132.60,131.29,129.19,126.39,117.50,103.40,55.85,21.83$. These spectroscopic data correspond to the reported data in reference [36].

(4-Fluorophenyl)(6-methoxybenzo[d]thiazol-2-yl)methanone (3bc): Yellow solid; yield 75\% (63 mg); m.p.: $167-169{ }^{\circ} \mathrm{C} ;{ }^{1} \mathrm{H}$ NMR $\left(500 \mathrm{MHz}, \mathrm{CDCl}_{3}\right) \delta 8.67(\mathrm{dd}, J=8.5,5.5 \mathrm{~Hz}, 2 \mathrm{H}), 8.11(\mathrm{~d}$, $J=9.5 \mathrm{~Hz}, 1 \mathrm{H}), 7.43(\mathrm{~d}, J=2.0 \mathrm{~Hz}, 1 \mathrm{H}), 7.27-7.20(\mathrm{~m}, 3 \mathrm{H}), 3.94(\mathrm{~s}, 3 \mathrm{H}) ;{ }^{13} \mathrm{C}$ NMR $(125 \mathrm{MHz}$, $\left.\mathrm{CDCl}_{3}\right) \delta 183.35,167.27,165.23,164.50,159.84,148.48,139.10,134.02(\mathrm{~d}, J=8.8 \mathrm{~Hz}), 126.43$, $117.71,115.64(\mathrm{~d}, J=22.5 \mathrm{~Hz}), 103.41,55.89$. These spectroscopic data correspond to the reported data in reference [36].

(4-Chlorophenyl)(6-methoxybenzo[d]thiazol-2-yl)methanone (3bd): Yellow solid; yield 76\% (69 mg); m.p.: $195-197^{\circ} \mathrm{C} ;{ }^{1} \mathrm{H}$ NMR $\left(500 \mathrm{MHz}, \mathrm{CDCl}_{3}\right) \delta 88.58-8.53(\mathrm{~m}, 2 \mathrm{H}), 8.12(\mathrm{~d}$, $J=9.0 \mathrm{~Hz}, 1 \mathrm{H}), 7.56-7.53(\mathrm{~m}, 2 \mathrm{H}), 7.43(\mathrm{~d}, J=2.5 \mathrm{~Hz}, 1 \mathrm{H}), 7.21(\mathrm{dd}, J=9.0,2.5 \mathrm{~Hz}, 1 \mathrm{H})$, $3.95(\mathrm{~s}, 3 \mathrm{H}) ;{ }^{13} \mathrm{C}$ NMR $\left(125 \mathrm{MHz}, \mathrm{CDCl}_{3}\right) \delta 181.31,164.28,159.91,148.48,140.82,140.34$, $133.29,132.60,128.79,126.50,117.79,103.39,55.88$. These spectroscopic data correspond to the reported data in reference [36].

(4-Bromophenyl)(6-methoxybenzo[d]thiazol-2-yl)methanone (3be): Yellow solid; yield 90\% (93 mg); m.p.:184-186 ${ }^{\circ} \mathrm{C} ;{ }^{1} \mathrm{H} \mathrm{NMR}\left(500 \mathrm{MHz}, \mathrm{CDCl}_{3}\right) \delta 8.09-8.04(\mathrm{~m}, 2 \mathrm{H}), 7.97(\mathrm{~d}, J=9.0 \mathrm{~Hz}$ $1 \mathrm{H}), 7.52-7.46(\mathrm{~m}, 3 \mathrm{H}), 7.37(\mathrm{~d}, J=2.5 \mathrm{~Hz}, 1 \mathrm{H}), 7.11(\mathrm{dd}, J=9.0,2.5 \mathrm{~Hz}, 1 \mathrm{H}), 3.91(\mathrm{~s}, 3 \mathrm{H})$; ${ }^{13} \mathrm{C}$ NMR $\left(125 \mathrm{MHz}, \mathrm{CDCl}_{3}\right) \delta 181.47,164.14,159.94,148.61,139.22,132.68,131.79,128.60$, $126.52,117.81,103.39,55.88$. These spectroscopic data correspond to the reported data in reference [27].

(4-Iodophenyl)(6-methoxybenzo[d] thiazol-2-yl)methanone (3bf): Yellow solid; yield 72\% (85 mg); m.p.: $152-153^{\circ} \mathrm{C} ;{ }^{1} \mathrm{H}$ NMR $\left(500 \mathrm{MHz}, \mathrm{CDCl}_{3}\right) \delta 8.31-8.26(\mathrm{~m}, 2 \mathrm{H}), 8.11(\mathrm{~d}, J=9.0 \mathrm{~Hz}, 1 \mathrm{H})$, 7.96-7.93 (m, 2H), $7.43(\mathrm{~d}, J=2.0 \mathrm{~Hz}, 1 \mathrm{H}), 7.21(\mathrm{dd}, J=9.0,2.5 \mathrm{~Hz}, 1 \mathrm{H}), 3.95(\mathrm{~s}, 3 \mathrm{H}) ;{ }^{13} \mathrm{C}$ NMR $\left(125 \mathrm{MHz}, \mathrm{CDCl}_{3}\right) \delta 184.38,163.07,159.92,148.62,145.50,144.12,142.39,137.79,132.47$, $126.52,117.80,103.39,99.99,55.88$. ESI-HRMS calcd $\mathrm{C}_{15} \mathrm{H}_{10} \mathrm{INO}_{2} \mathrm{~S}[\mathrm{M}+\mathrm{H}]^{+}$359.9555; found, 359.9576 .

(4-Butylphenyl)(6-methoxybenzo[d]thiazol-2-yl)methanone (3bg): Yellow solid; yield 92\% (90 mg); m.p.: $100-102{ }^{\circ} \mathrm{C} ;{ }^{1} \mathrm{H}$ NMR $\left(500 \mathrm{MHz}, \mathrm{CDCl}_{3}\right) \delta 8.49(\mathrm{~d}, J=8.0 \mathrm{~Hz}, 2 \mathrm{H}), 8.11(\mathrm{~d}, J=9.5 \mathrm{~Hz}$, $1 \mathrm{H}), 7.42(\mathrm{~d}, J=2.5 \mathrm{~Hz}, 1 \mathrm{H}), 7.37(\mathrm{~d}, J=8.5 \mathrm{~Hz}, 2 \mathrm{H}), 7.21-7.17(\mathrm{~m}, 1 \mathrm{H}), 3.93(\mathrm{~s}, 3 \mathrm{H}), 2.74-2.71$ (m, 2H), 1.69-1.65 (m, 2H), 1.43-1.38 (m, 2H), 0.98-0.94 (m, 3H); ${ }^{13} \mathrm{C}$ NMR $(125 \mathrm{MHz}$, $\left.\mathrm{CDCl}_{3}\right) \delta 184.71,165.02,159.67,149.60,139.02,132.77,131.32,129.07,128.57,126.37,117.48$, $103.42,55.83,35.85,33.17,22.34,13.90$. ESI-HRMS calcd $\mathrm{C}_{19} \mathrm{H}_{20} \mathrm{NO}_{2} \mathrm{~S}[\mathrm{M}+\mathrm{H}]^{+} 326.1215$; found, 326.1220 .

(6-Methoxybenzo[d]thiazol-2-yl)(4-methoxyphenyl)methanone (3bh): Yellow solid; yield 86\% (77 mg); m.p.: $147-149{ }^{\circ} \mathrm{C} ;{ }^{1} \mathrm{H}$ NMR $\left(500 \mathrm{MHz}, \mathrm{CDCl}_{3}\right) \delta 8.65(\mathrm{~d}, J=9.0 \mathrm{~Hz}, 2 \mathrm{H}), 8.11$ $(\mathrm{d}, J=9.0 \mathrm{~Hz}, 1 \mathrm{H}), 7.43(\mathrm{~d}, J=2.5 \mathrm{~Hz}, 1 \mathrm{H}), 7.19(\mathrm{~d}, J=9.0 \mathrm{~Hz}, 1 \mathrm{H}), 7.05(\mathrm{~d}, J=9.0 \mathrm{~Hz}$, 2H), 3.94 (s, 6H).; ${ }^{13} \mathrm{C}$ NMR (125 MHz, $\left.\mathrm{CDCl}_{3}\right) \delta 183.26,165.43,164.23,159.59,148.55$, 
$138.92,133.70,127.99,126.25,117.39,113.82,103.44,55.84,55.53$. These spectroscopic data correspond to the reported data in reference [27].

(6-Methoxybenzo[d]thiazol-2-yl)(4-nitrophenyl)methanone (3bi): Yellow solid; yield 75\% (71 mg); m.p.: $152-153{ }^{\circ} \mathrm{C} ;{ }^{1} \mathrm{H}$ NMR $\left(500 \mathrm{MHz}, \mathrm{CDCl}_{3}\right) \delta 8.73(\mathrm{~d}, J=9.0 \mathrm{~Hz}, 2 \mathrm{H}), 8.40(\mathrm{~d}, J=9.0 \mathrm{~Hz}$, $2 \mathrm{H}), 8.13(\mathrm{~d}, J=9.5 \mathrm{~Hz}, 1 \mathrm{H}), 8.02(\mathrm{~d}, J=9.0 \mathrm{~Hz}, 1 \mathrm{H}), 7.44(\mathrm{~d}, J=2.5 \mathrm{~Hz}, 1 \mathrm{H}), 3.96(\mathrm{~s}, 3 \mathrm{H})$; ${ }^{13} \mathrm{C}$ NMR $\left(125 \mathrm{MHz}, \mathrm{CDCl}_{3}\right) \delta 183.58,163.29,160.30,148.69,139.35,137.18,137.05,132.16$, $127.81,126.73,123.42,116.60,103.40,55.86$. These spectroscopic data correspond to the reported data in reference [47].

(5-Chlorobenzo[d]thiazol-2-yl)(phenyl)methanone (3bj): Yellow solid; yield 84\% (68 mg); m.p. $=133-135{ }^{\circ} \mathrm{C} ;{ }^{1} \mathrm{H}$ NMR $\left(500 \mathrm{MHz}, \mathrm{CDCl}_{3}\right) \delta 8.59-8.55(\mathrm{~m}, 2 \mathrm{H}), 8.26(\mathrm{~d}, J=2.0 \mathrm{~Hz}, 1 \mathrm{H})$, $7.95(\mathrm{~d}, J=8.5 \mathrm{~Hz}, 1 \mathrm{H}), 7.70(\mathrm{t}, J=7.5 \mathrm{~Hz}, 1 \mathrm{H}), 7.60-7.53(\mathrm{~m}, 3 \mathrm{H}) ;{ }^{13} \mathrm{C} \mathrm{NMR}(125 \mathrm{MHz}$, $\left.\mathrm{CDCl}_{3}\right) \delta 184.94,168.98,154.66,135.26,134.72,134.14,133.06,131.33,128.59,128.26,125.28$, 122.98. These spectroscopic data correspond to the reported data in reference [27].

(5-Chlorobenzo[d]thiazol-2-yl)(p-tolyl)methanone (3bk): Yellow solid; yield 76\% (65 mg); m.p.: 92-93 ${ }^{\circ} \mathrm{C} ;{ }^{1} \mathrm{H}$ NMR $\left(500 \mathrm{MHz}, \mathrm{CDCl}_{3}\right) \delta 8.48(\mathrm{~d}, J=8.5 \mathrm{~Hz}, 2 \mathrm{H}), 8.22(\mathrm{~d}, J=1.5 \mathrm{~Hz}, 1 \mathrm{H})$, $7.92(\mathrm{~d}, J=8.5 \mathrm{~Hz}, 1 \mathrm{H}), 7.50(\mathrm{dd}, J=8.5,1.5 \mathrm{~Hz}, 1 \mathrm{H}), 7.36(\mathrm{~d}, J=8.0 \mathrm{~Hz}, 2 \mathrm{H}), 2.48(\mathrm{~s}, 3 \mathrm{H})$; ${ }^{13} \mathrm{C}$ NMR $\left(125 \mathrm{MHz}, \mathrm{CDCl}_{3}\right) \delta 184.40,169.32,154.64,145.28,135.19,132.93,132.14,131.44$, $129.31,128.07,125.18,122.91,21.84$. These spectroscopic data correspond to the reported data in reference [27].

(5-Chlorobenzo[d]thiazol-2-yl)(4-fluorophenyl)methanone (3bl): Yellow solid; yield 73\% (63 mg); m.p.: $142-144{ }^{\circ} \mathrm{C} ;{ }^{1} \mathrm{H}$ NMR $\left(500 \mathrm{MHz}, \mathrm{CDCl}_{3}\right) \delta 8.75-8.62(\mathrm{~m}, 2 \mathrm{H}), 8.25(\mathrm{~d}, J=2.0 \mathrm{~Hz}, 1 \mathrm{H})$, $7.96(\mathrm{~d}, J=8.5 \mathrm{~Hz}, 1 \mathrm{H}), 7.55(\mathrm{dd}, J=8.5,2.0 \mathrm{~Hz}, 1 \mathrm{H}), 7.25(\mathrm{~d}, J=8.5 \mathrm{~Hz}, 2 \mathrm{H}) ;{ }^{13} \mathrm{C} \mathrm{NMR}$ $\left(125 \mathrm{MHz} \mathrm{CDCl}_{3}\right) \delta 183.15,168.89,165.55,154.60,135.24,134.25$ (d, J = 8.8 Hz), 133.15, $128.35,125.25,122.99,115.85(\mathrm{~d}, J=22.5 \mathrm{~Hz})$. These spectroscopic data correspond to the reported data in reference [48].

(5-Chlorobenzo[d] thiazol-2-yl)(4-chlorophenyl)methanone (3bm): Yellow solid; yield 78\% (72 mg); m.p.: ${ }^{185-187 ~}{ }^{\circ} \mathrm{C} ;{ }^{1} \mathrm{H}$ NMR $\left(500 \mathrm{MHz}, \mathrm{CDCl}_{3}\right) \delta 8.60-8.53(\mathrm{~m}, 3 \mathrm{H}), 8.25(\mathrm{~d}, \mathrm{~J}=2.0 \mathrm{~Hz}$, $1 \mathrm{H}), 7.96(\mathrm{~d}, J=8.5 \mathrm{~Hz}, 1 \mathrm{H}), 7.57-7.54(\mathrm{~m}, 3 \mathrm{H}) ;{ }^{13} \mathrm{C} \mathrm{NMR}\left(125 \mathrm{MHz}, \mathrm{CDCl}_{3}\right) \delta 183.61$, $168.66,154.60,140.95,135.29,133.21,133.00,132.76,128.97,128.44,125.31,123.01$. These spectroscopic data correspond to the reported data in reference [26].

(4-Bromophenyl)(5-chlorobenzo[d]thiazol-2-yl)methanone (3bn): Yellow solid; yield 80\% (84 mg); m.p.: $184-186{ }^{\circ} \mathrm{C}^{1}{ }^{1} \mathrm{H}$ NMR $\left(500 \mathrm{MHz}, \mathrm{CDCl}_{3}\right) \delta 8.51-8.44(\mathrm{~m}, 1 \mathrm{H}), 8.25(\mathrm{~d}, J=2.0 \mathrm{~Hz}, 1 \mathrm{H})$, $7.96(\mathrm{~d}, J=9.0 \mathrm{~Hz}, 0 \mathrm{H}), 7.74-7.71(\mathrm{~m}, 1 \mathrm{H}), 7.55(\mathrm{dd}, J=8.5,2.0 \mathrm{~Hz}, 1 \mathrm{H}) ;{ }^{13} \mathrm{C} \mathrm{NMR}(125 \mathrm{MHz}$, $\left.\mathrm{CDCl}_{3}\right) \delta 183.83,168.60,154.59,135.29,133.41,133.22,132.79,131.96,129.83,128.45,125.31$, 123.00. These spectroscopic data correspond to the reported data in reference [27].

(5-Chlorobenzo[d]thiazol-2-yl)(4-iodophenyl)methanone (3bo): Yellow solid; yield 73\% (87 mg); m.p.: $182-184{ }^{\circ} \mathrm{C} ;{ }^{1} \mathrm{H} \mathrm{NMR}\left(500 \mathrm{MHz}, \mathrm{CDCl}_{3}\right) \delta 8.32-8.30(\mathrm{~m}, 2 \mathrm{H}), 8.25(\mathrm{~d}, J=2.0 \mathrm{~Hz}, 1 \mathrm{H})$, $7.95(\mathrm{~d}, J=2.0 \mathrm{~Hz}, 1 \mathrm{H}), 7.70(\mathrm{~d}, J=4.5 \mathrm{~Hz}, 2 \mathrm{H}), 7.55(\mathrm{dd}, J=8.5,2.0 \mathrm{~Hz}, 1 \mathrm{H}) ;{ }^{13} \mathrm{C} \mathrm{NMR}$ $\left(125 \mathrm{MHz} \mathrm{CDCl}_{3}\right) \delta 183.45,169.19,154.55,135.26,133.91,133.17,132.71,131.90,130.93$, 128.42, 125.28, 122.99. ESI-HRMS calcd $\mathrm{C}_{14} \mathrm{H}_{8} \mathrm{CIINOS}[\mathrm{M}+\mathrm{H}]^{+}$399.9060; found, 399.9080 .

[1,1'-Biphenyl]-4-yl(5-chlorobenzo[d]thiazol-2-yl)methanone (3bp): Yellow solid; yield 78\% (81 mg); m.p.: ${ }^{159-161 ~}{ }^{\circ} \mathrm{C} ;{ }^{1} \mathrm{H}$ NMR $\left(500 \mathrm{MHz}, \mathrm{CDCl}_{3}\right) \delta 8.68-8.63(\mathrm{~m}, 2 \mathrm{H}), 8.27(\mathrm{~d}, J=1.5 \mathrm{~Hz}$, $1 \mathrm{H}), 7.95(\mathrm{~d}, J=8.5 \mathrm{~Hz}, 1 \mathrm{H}), 7.81-7.78(\mathrm{~m}, 2 \mathrm{H}), 7.72-7.68(\mathrm{~m}, 2 \mathrm{H}), 7.55-7.50(\mathrm{~m}, 3 \mathrm{H})$, 7.47-7.42 (m, 1H); ${ }^{13} \mathrm{C}$ NMR $\left(125 \mathrm{MHz}, \mathrm{CDCl}_{3}\right) \delta 184.26,169.17,154.64,146.77,139.77$, $135.23,133.35,133.02,131.91,129.01,128.46,128.19,127.36,127.19,125.23,122.95$. ESIHRMS calcd $\mathrm{C}_{20} \mathrm{H}_{13} \mathrm{CINOS}[\mathrm{M}+\mathrm{H}]^{+}$350.0406; found, 350.0410.

(6-Nitrobenzo[d]thiazol-2-yl)(phenyl)methanone (3bq): Yellow solid; yield 87\% (74 mg); m.p.: 159-161 ${ }^{\circ} \mathrm{C} ;{ }^{1} \mathrm{H} \mathrm{NMR}\left(500 \mathrm{MHz}, \mathrm{CDCl}_{3}\right) \delta 8.98(\mathrm{~d}, J=2.0 \mathrm{~Hz}, 1 \mathrm{H}), 8.61(\mathrm{dd}, J=8.0,0.5 \mathrm{~Hz}$, $2 \mathrm{H}), 8.47(\mathrm{dd}, J=9.0,2.5 \mathrm{~Hz}, 1 \mathrm{H}), 8.39(\mathrm{~d}, J=9.0 \mathrm{~Hz}, 1 \mathrm{H}), 7.74(\mathrm{t}, J=7.5 \mathrm{~Hz}, 1 \mathrm{H}), 7.61(\mathrm{t}$, $J=8.0 \mathrm{~Hz}, 2 \mathrm{H}) ;{ }^{13} \mathrm{C} \mathrm{NMR}\left(125 \mathrm{MHz}, \mathrm{CDCl}_{3}\right) \delta 191.14,172.44,157.19,146.61,137.14,134.59$, 
$134.26,131.41,128.75,126.24,122.08,118.86$. These spectroscopic data correspond to the reported data in reference [36].

2-Benzoylbenzo[d] thiazole-6-carbonitrile (3br): Yellow solid; yield 80\% (63 mg); m.p.: 140-142 ${ }^{\circ} \mathrm{C}$; ${ }^{1} \mathrm{H} \mathrm{NMR}\left(500 \mathrm{MHz}, \mathrm{CDCl}_{3}\right) \delta 8.59(\mathrm{~d}, J=7.0 \mathrm{~Hz}, 2 \mathrm{H}), 8.40(\mathrm{~d}, J=1.0 \mathrm{~Hz}, 1 \mathrm{H}), 8.35$ $(\mathrm{d}, J=8.5 \mathrm{~Hz}, 1 \mathrm{H}), 7.84(\mathrm{dd}, J=8.5,1.5 \mathrm{~Hz}, 1 \mathrm{H}), 7.73(\mathrm{t}, J=7.5 \mathrm{~Hz}, 1 \mathrm{H}), 7.60(\mathrm{t}, J=8.0 \mathrm{~Hz}$, $2 \mathrm{H}) ;{ }^{13} \mathrm{C} \mathrm{NMR}\left(125 \mathrm{MHz}, \mathrm{CDCl}_{3}\right) \delta 171.14,155.94,134.51,134.34,131.38,129.67,128.72$, $127.26,126.54,118.30,111.20$. These spectroscopic data correspond to the reported data in reference [36].

Supplementary Materials: The following are available online.

Author Contributions: Methodology, X.-T.S. and J.-Q.W.; validation, Z.H. and L.-L.Z.; data curation, X.-T.S. and Z.-G.H.; writing_original draft, X.-T.S.; writing—review and editing, J.-Q.W.; supervision, J.-Q.W.; project administration, J.-Q.W.; funding acquisition, J.-Q.W. All authors have read and agreed to the published version of the manuscript.

Funding: This research was financially supported by the Key Research and Development Program of Zhejiang Province (Grant No. 2022C03016) and the Zhejiang Provincial Natural Science Foundation of China (Grant No. LY17C140003).

Institutional Review Board Statement: Not applicable.

Informed Consent Statement: Not applicable.

Data Availability Statement: The data presented in this study is available in the article or supporting information.

Conflicts of Interest: The authors declare no conflict of interest.

Sample Availability: Samples of the compounds are available from the authors.

\section{References}

1. Blunt, C.E.; Nawrat, C.C.; LeBozec, L.; Liutkus, M.; Liu, Y.; Lewis, W.; Moody, C.J. Oxidative routes to the heterocyclic cores of benzothiazole natural products. Synlett 2016, 27, 37-40.

2. Sumit; Kumar, A.; Mishra, A.K. Advancement in pharmacological activities of benzothiazole and its derivatives: An up to date review. Mini-Rev. Med. Chem. 2021, 21, 314-335.

3. Ballari, M.S.; Cano, N.H.; Wunderlin, D.A.; Feresin, G.E.; Santiago, A.N. One-pot sequential synthesis and antifungal activity of 2-(benzylsulfonyl)benzothiazole derivatives. RSC Adv. 2019, 9, 29405-29413. [CrossRef]

4. Bhat, M.; Belagali, S.L. Structural activity relationship and importance of benzothiazole derivatives in medicinal chemistry: A comprehensive review. Mini-Rev. Org. Chem. 2020, 17, 323-350. [CrossRef]

5. Dhumal, S.T.; Deshmukh, A.R.; Kharat, K.R.; Sathe, B.R.; Chavan, S.S.; Mane, R.A. Copper fluorapatite assisted synthesis of new 1,2,3-triazoles bearing a benzothiazolyl moiety and their antibacterial and anticancer activities. New J. Chem. 2019, 43, 7663-7673. [CrossRef]

6. El-Mekabaty, A.; Sofan, M.A.; Hasel, A.M.; Said, S.B. Concise synthesis of some new benzothiazole-based heterocycles as probable anticancer and antioxidant agents. ChemistrySelect 2021, 6, 2569-2575. [CrossRef]

7. Hori, N.; Tsukamoto, G.; Imamura, A.; Ohashi, M.; Saito, T.; Yoshino, K. Novel disease-modifying antirheumatic drugs.1. synthesis and antiarthritic activity of 2-(4-methylphenyl)benzothiazoles. Chem. Pharm. Bull. 1992, 40, 2387-2390. [CrossRef]

8. Haroun, M.; Tratrat, C.; Petrou, A.; Geronikaki, A.; Ivanov, M.; Ciric, A.; Sokovic, M. 2-Aryl-3-(6-trifluoromethoxy)benzo[d]thiazolebased thiazolidinone hybrids as potential anti-infective agents: Synthesis, biological evaluation and molecular docking studies. Bioorg. Med. Chem. Lett. 2021, 32, 127718. [CrossRef]

9. Pal, N.; Arya, A.K. An efficient and facile synthesis of $\mathrm{Zn}(\mathrm{II})$ complexes with 2-substituted benzothiazoles and glycine- and alanine-based ligands having antifungal and antibacterial activities. Res. Chem. Intermed. 2013, 39, 553-560. [CrossRef]

10. Gilani, S.J.; Hassan, M.Z.; Imam, S.S.; Kala, C.; Dixit, S.P. Novel benzothiazole hydrazine carboxamide hybrid scaffolds as potential in vitro GABA AT enzyme inhibitors: Synthesis, molecular docking and antiepileptic evaluation. Bioorg. Med. Chem. Lett. 2019, 29, 1825-1830. [CrossRef]

11. Tang, X.; Wang, Z.B.; Zhong, X.M.; Wang, X.B.; Chen, L.J.; He, M.; Xue, W. Synthesis and biological activities of benzothiazole derivatives bearing a 1,3,4-thiadiazole moiety. Phosphorus Sulfur Silicon Relat. Elem. 2019, 194, 241-248. [CrossRef]

12. Miralinaghi, P.; Schmitt, C.; Hartmann, R.W.; Frotscher, M.; Engel, M. 6-Hydroxybenzothiophene ketones: Potent inhibitors of 17 beta-hydroxysteroid dehydrogenase type 1 (17 beta-HSD1) owing to favorable molecule geometry and conformational preorganization. ChemMedChem 2014, 9, 2294-2308. [CrossRef] 
13. Spadaro, A.; Negri, M.; Marchais-Oberwinkler, S.; Bey, E.; Frotscher, M. Hydroxybenzothiazoles as new nonsteroidal inhibitors of 17 beta-hydroxysteroid dehydrogenase Type 1 (17 beta-HSD1). PLoS ONE 2012, 7, e292522012. [CrossRef]

14. Spadaro, A.; Frotscher, M.; Hartmann, R.W. Optimization of hydroxybenzothiazoles as novel potent and selective inhibitors of 17 beta-HSD1. J. Med. Chem. 2012, 55, 2469-2473. [CrossRef] [PubMed]

15. Komiya, M.; Asano, S.; Koike, N.; Koga, E.; Igarashi, J.; Nakatani, S.; Isobe, Y. Synthesis of novel benzo-fused heteroaryl derivatives as $\mathrm{Ca}^{2+} /$ Calmodulin-dependent protein kinase II inhibitors. Chem. Pharm. Bull. 2013, 61, 1094-1097. [CrossRef]

16. Myllymaki, M.J.; Saario, S.M.; Kataja, A.O.; Castillo-Melendez, J.A.; Nevalainen, T.; Juvonen, R.O.; Jarvinen, T.; Koskinen, A.M.P. Design, synthesis, and in vitro evaluation of carbamate derivatives of 2-benzoxazolyl- and 2-benzothiazolyl-(3-hydroxyphenyl)methanones as novel fatty acid amide hydrolase inhibitors. J. Med. Chem. 2007, 50, 4236-4242. [CrossRef] [PubMed]

17. Tang, G.Z.; Nikolovska-Coleska, Z.; Qiu, S.; Yang, C.Y.; Guo, J.; Wang, S.M. Acylpyrogallols as inhibitors of antiapoptotic Bcl-2 proteins. J. Med. Chem. 2008, 51, 717-720. [CrossRef] [PubMed]

18. Chen, J.J.; Li, C.M.; Wang, J.; Ahn, S.; Wang, Z.; Lu, Y.; Dalton, J.T.; Miller, D.D.; Li, W. Synthesis and antiproliferative activity of novel 2-aryl-4-benzoyl-imidazole derivatives targeting tubulin polymerization. Bioorg. Med. Chem. 2011, 19, 4782-4795. [CrossRef]

19. Hu, E.; Kunz, R.K.; Chen, N.; Rumfelt, S.; Siegmund, A.; Andrews, K.; Chmait, S.; Zhao, S.; Davis, C.; Chen, H.; et al. Design, optimization, and biological evaluation of novel keto-benzimidazoles as potent and selective inhibitors of phosphodiesterase $10 \mathrm{~A}$ (PDE10A). J. Med. Chem. 2013, 56, 8781-8792. [CrossRef]

20. Zhu, Y.P.; Jia, F.C.; Liu, M.C.; Wu, A.X.A. Multipathway coupled domino strategy: Metal-free oxidative cyclization for one-pot synthesis of 2-acylbenzothiazoles from multiform substrates. Org. Lett. 2012, 14, 4414-4417. [CrossRef]

21. Fan, X.S.; He, Y.; Zhang, X.Y.; Guo, S.G.; Wang, Y.Y. Synthesis of heteroaryl ketones via tandem reaction of 1,1-dibromoethenes. Tetrahedron 2011, 67, 6369-6374. [CrossRef]

22. Meng, X.; Bi, X.R.; Yu, C.Y.; Chen, G.X.; Chen, B.H.; Jing, Z.Q.; Zhao, P.Q. Ball-milling synthesized hydrotalcite supported $\mathrm{Cu}-\mathrm{Mn}$ mixed oxide under solvent-free conditions: An active catalyst for aerobic oxidative synthesis of 2-acylbenzothiazoles and quinoxalines. Green Chem. 2018, 20, 4638-4644. [CrossRef]

23. Huynh, T.V.; Doan, K.V.; Luong, N.T.K.; Nguyen, D.T.P.; Doan, S.H.; Nguyen, T.T.; Phan, N.T.S. New synthesis of 2aroylbenzothiazoles via metal-free domino transformations of anilines, acetophenones, and elemental sulfur. RSC Adv. 2020, 10, 18423-18433. [CrossRef]

24. Liu, S.W.; Chen, R.; Chen, H.; Deng, G.J. Iron-catalyzed 2-acylbenzothiazole formation from aryl ketones and benzothiazoles using oxygen as oxidant. Tetrahedron Lett. 2013, 54, 3838-3841. [CrossRef]

25. Wang, J.; Zhang, X.Z.; Chen, S.Y.; Yu, X.Q. Iron-catalyzed arylation or aroylation of benzothiazoles with benzylic alcohols and aryl ketones. Tetrahedron 2014, 70, 245-250. [CrossRef]

26. Feng, Q.; Song, Q.L. Chemoselective copper-catalyzed acylation of benzothiazoles with aryl methyl ketones. Adv. Synth. Catal. 2014, 356, 2445-2452. [CrossRef]

27. Wang, B.; Zhang, Q.W.; Guo, Z.Q.; Ablajan, K. Iodine- and TBHP-promoted acylation of benzothiazoles under metal-free conditions. Synthesis 2020, 52, 3058-3064.

28. Hua, M.; Wang, C.Q.; Liu, Q.X.; Chen, D.Y.; Fu, H.; Zhou, H.F. Silver-mediated 2-arylation/alkylation/acylation of benzothiazoles with aldehydes in water. Heterocycles 2018, 96, 1226-1237.

29. Yang, K.; Zhang, C.; Wang, P.; Zhang, Y.; Ge, H.B. Nickel-catalyzed decarboxylative acylation of heteroarenes by $\mathrm{sp}^{2} \mathrm{C}-\mathrm{H}$ functionalization. Chem.-Eur. J. 2014, 20, 7241-7244. [CrossRef]

30. Yang, K.; Chen, X.Y.; Wang, Y.Q.; Li, W.Q.; Kadi, A.A.; Fun, H.K.; Sun, H.; Zhang, Y.; Li, G.G.; Lu, H.J. Cobalt-catalyzed decarboxylative 2-benzoylation of oxazoles and thiazoles with alpha-oxocarboxylic acids. J. Org. Chem. 2015, 80, 11065-11072. [CrossRef]

31. Huang, T.H.; Wu, X.; Yu, Y.B.; An, L.; Yin, X.X. A convenient synthesis of 2-acyl benzothiazoles/thiazoles from benzothiazole/thiazole and $N, N^{\prime}$-carbonyldiimidazole activated carboxylic acids. Tetrahedron Lett. 2019, 60, 1667-1670. [CrossRef]

32. Lassalas, P.; Marsais, F.; Hoarau, C. DMAP-catalyzed regel-type direct C-2 (hetero)aroylation of oxazoles and thiazoles derivatives with acid chlorides. Synlett 2013, 24, 2233-2240. [CrossRef]

33. Wirth, T. Hypervalent iodine chemistry in synthesis: Scope and new directions. Angew. Chem. Int. Edit. 2005, 44, 3656-3665. [CrossRef] [PubMed]

34. Richardson, R.D.; Wirth, T. Hypervalent iodine goes catalytic. Angew. Chem. Int. Edit. 2006, 45, 4402-4404. [CrossRef]

35. Merritt, E.A.; Olofsson, B. Alpha-functionalization of carbonyl compounds using hypervalent iodine reagents. Synthesis 2011, 517-538. [CrossRef]

36. Lu, Q.; Sun, X.T.; Kong, Y.L.; Liu, J.C.; Chen, B.; Weng, J.Q. C2-arylacylation of 2H-benzothiazoles with methyl arenes via selectfluor oxidation. Tetrahedron Lett. 2021, 75, 153184. [CrossRef]

37. Weng, J.Q.; Xu, W.X.; Dai, X.Q.; Zhang, J.H.; Liu, X.H. Alkylation reactions of benzothiazoles with $N, N$-dimethylamides catalyzed by the two-component system under visible light. Tetrahedron Lett. 2019, 60, 390-396. [CrossRef]

38. Xu, W.X.; Dai, X.Q.; Weng, J.Q. $\mathrm{K}_{2} \mathrm{~S}_{2} \mathrm{O}_{8}$-mediated hydroxyalkylation of benzothiazoles with alcohols in aqueous solution. ACS Omega 2019, 4, 11285-11292. [CrossRef] [PubMed]

39. Kong, Y.L.; Xu, W.X.; Liu, X.H.; Weng, J.Q. Visible light-induced hydroxyalkylation of $2 H$-benzothiazoles with alcohols via selectfluor oxidation. Chin. Chem. Lett. 2020, 31, 3245-3249. [CrossRef] 
40. Xu, W.X.; Ye, F.X.; Liu, X.H.; Weng, J.Q. NCS/TBHP promoted C2 arylation of benzothiazoles with aldehydes in DMSO. Tetrahedron Lett. 2020, 61, 151807. [CrossRef]

41. Zhu, Y.P.; Liu, M.C.; Jia, F.C.; Yuan, J.J.; Gao, Q.H.; Lian, M.; Wu, A.X. Metal-free $\mathrm{sp}^{3}$ C-H bond dual-(het)arylation: $\mathrm{I}_{2}$-promoted domino process to construct 2,2-bisindolyl-1-arylethanones. Org. Lett. 2012, 14, 3392-3395. [CrossRef]

42. Ghorbani-Choghamarani, A.; Taherinia, Z. New revolution in biaryl synthesis: Transition metal-free C-C bond formation promoted by the mixture of 2-mercaptoethanol/KOH/DMSO. ChemistrySelect 2019, 4, 4735-4738. [CrossRef]

43. Matcha, K.; Antonchick, A.P. Metal-free cross-dehydrogenative coupling of heterocycles with aldehydes. Angezw. Chem. Int. Edit. 2013, 52, 2082-2086. [CrossRef] [PubMed]

44. Yang, Q.M.; Han, X.L.; Zhao, J.Q.; Zhang, H.Y.; Zhang, Y.C. Direct C3 Alkoxylation of Quinoxalin-2(1H)-ones with Alcohols via Cross-Dehydrogenative Coupling under Catalyst-Free Conditions. J. Org. Chem. 2019, 84, 11417-11424. [CrossRef] [PubMed]

45. Li, X.; Liu, C.Y.; Guo, S.X.; Wang, W.; Zhang, Y.Q. PIFA-mediated cross-dehydrogenative coupling of N-heteroarenes with cyclic ethers: Ethanol as an efficient promoter. Eur. J. Org. Chem. 2021, 2021, 411-421. [CrossRef]

46. Zhang, S.S.; Wang, S.W.; Leng, Y.T.; Wu, Y.J. One-pot synthesis of 2-acylbenzothiazoles from 2-aminobenzenethiols and arylacetonitriles via cyclization and sequential oxidation. Tetrahedron Lett. 2021, 79, 153300. [CrossRef]

47. Fukuda, K.; Hasegawa, T.; Kotani, T.; Muramoto, H.; Okamoto, K. Benzothiazole Derivatives. U.S. Patent 5,900,426, 4 May 1999.

48. Ma, R.C.; Ding, Y.X.; Chen, R.; Wang, Z.M.; Wang, L.; Ma, Y.M. Oxidant/solvent-controlled I 2 -catalyzed domino annulation for selective synthesis of 2-aroylbenzothiazoles and 2-arylbenzothiazoles under metal-free conditions. J. Org. Chem. 2021, 86, 310-321. [CrossRef] 